\title{
Should We Be Quick To Dismiss Non-Sphincter-Sparing Surgery for Fistula-in-Ano? An Analysis of Long-Term Outcomes
}

Corrado De Marco, Maria Abou-Khalil, Nancy Morin, Carol-Ann Vasilevsky, Julio Faria, Philip Gordon, Gabriela

Ghitulescu, Marylise Boutros

Division of Colon and Rectal Surgery, Sir Mortimer B. Davis Jewish General Hospital, Montreal, QC, Canada

\section{INTRODUCTION}

Fistulotomy is considered the gold-standard treatment for fistula-in-ano

- Reported rates of fecal incontinence and fistula recurrence are low post-fistulotomy, but there exists a lack of long-term functional studies

- A desire to reduce the risk of long-term fecal incontinence has prompted the emergence of sphincter-sparing treatment techniques, despite their higher risk of recurrence

\section{PURPOSE}

The purpose of this study is to compare the long-term risks of fecal incontinence and recurrence following sphinctersparing and non-sphincter-sparing procedures for fistulain-ano

\section{METHODS}

All patients with fistula-in-ano managed operatively between 2000 and 2012 by colorectal surgeons at a tertiary center were included

- Patients with inflammatory bowel disease, pelvic radiation, and non-definitive treatment were excluded

- Medical records and operative reports were reviewed and patients were contacted by telephone to document fecal incontinence (FI) using CCF-FIS and FIQL questionnaire

- Fistulas were characterized by: type, location, branching, number of internal openings, classification (i.e., high or low), and primary or recurrent fistula

- Procedures were classified as sphincter-sparing (i.e., fibrin glue, anal plug, anorectal flap, LIFT) or non-sphinctersparing (i.e., fistulotomy, cutting seton)

- Outcomes of interest were fecal incontinence (defined as CCF-FIS $\geq 10$ ) as a primary outcome and recurrence of disease as a secondary outcome

\section{FIGURE 1:}

\section{PATIENT SELECTION}

All charts coded as fistula-inano managed operatively at the Jewish General Hospital between 2000-2012 assessed for eligibility, $\mathrm{n}=962$

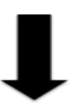

Patients meeting inclusion criteria whose charts were fully reviewed, $\mathbf{n}=\mathbf{3 3 8}$

\section{I}

Study group of patients whose charts were reviewed and who completed the long-term follow-up questionnaire, $\mathrm{n}=\mathbf{1 5 6}$

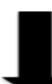

Study group of patients who underwent non-sphinctersparing procedures, $\mathbf{n}=119$
TABLE 4:

TABLE 1:

PATIENT CHARACTERISTICS

\begin{tabular}{|l|l|l|}
\hline & $\begin{array}{l}\text { Non-SSP } \\
(N=119)\end{array}$ & $\begin{array}{l}\text { SSP } \\
(N=37)\end{array}$ \\
\hline Age & $54.87( \pm 11.4)$ & $57.34( \pm 15.4)$ \\
\hline Sex & & \\
\hline Male & $95(79.8 \%)$ & $26(70.3 \%)$ \\
\hline Female & $24(20.2 \%)$ & $11(29.7 \%)$ \\
\hline ASA & & \\
\hline 1 & $62(52.1 \%)$ & $17(45.9 \%)$ \\
\hline 2 & $51(42.9 \%)$ & $16(43.2 \%)$ \\
\hline 3 & $6(5.0 \%)$ & $4(10.8 \%)$ \\
\hline 4 & $0(0.0 \%)$ & $0(0.0 \%)$ \\
\hline $\begin{array}{l}\text { BMI }(\mathrm{kg} / \\
\left.\mathrm{m}^{2}\right)\end{array}$ & $27.58( \pm 5.46)$ & $28.78( \pm 7.40)$ \\
\hline DMII & $8(6.72 \%)$ & $3(8.1 \%)$ \\
\hline Smoker & $25(21.0 \%)$ & $6(16.2 \%)$ \\
\hline $\begin{array}{l}\text { Vaginal } \\
\text { deliveries }\end{array}$ & $12(10.1 \%)$ & $4(10.8 \%)$ \\
\hline $\begin{array}{l}\text { Obstetrical } \\
\text { injuries }\end{array}$ & $7(5.9 \%)$ & $3(8.1 \%)$ \\
\hline $\begin{array}{l}\text { Recurrent } \\
\text { disease }\end{array}$ & $35(29.4 \%)$ & $2(5.4 \%)$ \\
\hline
\end{tabular}

\section{TABLE 3: OUTCOMES}

Recurrence and Fecal Incontinence (FI)

\begin{tabular}{|l|l|l|l|}
\hline & $\begin{array}{l}\text { Recurrence } \\
(N=45)\end{array}$ & $\begin{array}{l}\text { All FI } \\
(N=21)\end{array}$ & $\begin{array}{l}\text { CCF-FIS } \\
\geq 10\end{array}$ \\
\hline $\begin{array}{l}\text { SSP } \\
(N=37)\end{array}$ & $\begin{array}{l}22 \\
(59.4 \%)\end{array}$ & 0 & 0 \\
$(0.0 \%)$ & $(0.0 \%)$ \\
\hline $\begin{array}{l}\text { Non- } \\
\text { SSP } \\
(N=119)\end{array}$ & 23 & 21 & 2 \\
$(19.3 \%)$ & $(17.6 \%)$ & $(1.68 \%)$ \\
\hline
\end{tabular}

Fecal Incontinence Quality of Life:

Median FIQL scores (range 1-4; 4=not affected) were lifestyle

4.0 (2.0-4.0); coping 4.0 (1.3-4.0); depression 4.0 (1.3-4.0); embarrassment $4.0(1.3-4.0)$

\section{UNIVARIATE ANALYSIS OF RECURRENCE}

\begin{tabular}{|l|l|l|l|}
\hline Logistic Regression & Odds Ratio & $95 \% C I$ & p-value \\
\hline Variable & $\mathbf{6 . 1 2}$ & $\mathbf{2 . 7 5 - 1 3 . 6 0}$ & $<\mathbf{0 . 0 0 1}$ \\
\hline SSP & 1.01 & $0.96-1.07$ & 0.553 \\
\hline BMI & 0.98 & $0.95-1.00$ & 0.106 \\
\hline Age at first repair & 0.53 & $0.11-2.54$ & 0.425 \\
\hline DMII & 1.22 & $0.53-2.86$ & 0.640 \\
\hline Smoking & & & \\
\hline Secondary opening & Ref. & Ref. & Ref. \\
\hline \multicolumn{1}{|l|}{ Anterior } & $\mathbf{0 . 4 8}$ & $\mathbf{0 . 2 3 - 0 . 9 9}$ & $\mathbf{0 . 0 4 7}$ \\
\hline \multicolumn{1}{|c|}{ Posterior } & - & - & - \\
\hline \multicolumn{1}{|c|}{ Lateral } & 0.83 & $0.14-4.86$ & 0.839 \\
\hline Bilateral & 1.47 & $0.67-3.24$ & 0.335 \\
\hline Recurrent disease & 0.72 & $0.32-1.61$ & 0.421 \\
\hline Sex (male) & $\mathbf{3 . 7 3}$ & $\mathbf{1 . 6 0 - 6 . 4 1}$ & $\mathbf{0 . 0 0 2}$ \\
\hline Complex fistula & 1.05 & $0.93-1.40$ & 0.221 \\
\hline Follow-up time (years) & $\mathbf{4 . 2 2}$ & $\mathbf{2 . 0 0 - 8 . 9 4}$ & $<\mathbf{0 . 0 0 1}$ \\
\hline High fistula &
\end{tabular}

Median follow-up 9.1 years (6.5 years for non-sphinctersparing procedures vs. 12.6 years for sphincter-sparing procedures)

- Patients contacted to document fecal incontinence and to ensure that no recurrence treated
Study group of patients who underwent sphincter-sparing procedures, $\mathbf{n}=\mathbf{3 7}$

\section{STRENGTHS:} elsewhere be missed

- Significantly longer term follow-up than studies published to date

TABLE 5:

MULTIVARIATE ANALYSIS OF RECURRENCE Logistic Regression

\begin{tabular}{|l|l|l|l|}
\hline Variable & Odds Ratio & $95 \%$ CI & $p$-value \\
\hline SSP & $\mathbf{6 . 1 4}$ & $\mathbf{2 . 2 2}-\mathbf{1 7 . 0 2}$ & $<\mathbf{0 . 0 0 1}$ \\
\hline Complex fistula & 1.68 & $0.70-4.04$ & 0.243 \\
\hline BMI & 1.00 & $0.95-1.07$ & 0.772 \\
\hline Recurrent fistula & $\mathbf{2 . 6 2}$ & $\mathbf{1 . 0 2}-\mathbf{6 . 6 9}$ & $\mathbf{0 . 0 4 4}$ \\
\hline Posterior fistula & 0.84 & $0.38-1.88$ & 0.767 \\
\hline
\end{tabular}

\section{CONCLUSIONS}

Long-term rates of significant FI after non-sphinctersparing procedures were low and did not impact quality of life, indicating that these procedures remain a safe option with appropriate patient selection

The use of sphincter-sparing procedures for the definitive treatment of fistula-in-ano had significantly higher recurrence rates compared to non-sphinctersparing procedures

\section{LIMITATIONS:}

- Smaller number of SSPs due to increased use of the technique only being adopted in recent years (i.e., outside of study range) 